\title{
AN EPIDEMIC MODEL WITH DENSITY DEPENDENT PARAMETERS AND VACCINATION
}

\author{
Q.J.A. KHAN and B.S. BHATT \\ Department of Mathematics and Computing \\ College of Science \\ Sultan Qaboos University \\ P O Box 36, Postal Code 123 Al-Khod \\ Muscat, SULTANATE OF OMAN
}

(Received July 29,1993 and in revised form December 28, 1994)

\begin{abstract}
A model originally suggested by Greenhalgh [12] and later modified by that same author $[13,14]$ is considered under the assumption that the transmission coefficient is inversely proportional to the total population size The purpose of this study is to see the effect of this density dependent transmission coefficient on the stability criteria for the equilibrium of the model equations. It is found that Greenhalgh's results are still valid
\end{abstract}

KEY WORDS AND PHRASES. Epidemic model, density-dependent death rate and transmission coefficient, vaccination, stability

1992 AMS SUBJECT CLASSIFICATION CODES. 92B05

\section{INTRODUCTION.}

This paper uses a relatively simple deterministic mathematical model to describe infectious diseases like measles, rubella, chickenpox and mumps The book by Bailey [4] describes much of the background in the area of epidemic models up to 1975. We are interested in looking at a model where the parameters which describe the transmission of the disease and the death rate of individuals are dependent on the number of individuals in the population. We also take into account the fact that infected individuals suffer a higher death rate than other individuals because they have the disease Some models for a population with a density dependent death rate have been studied by Nisbet and Gurney [20] Anderson [1] has analyzed an epidemic model with birth and death in which infected individuals suffer a higher death rate than other individuals. He considers the death rate of individuals to be density independent Dietz and Schenzle [10] and Mollison [19] considered the transmission coefficient to be dependent on population density Anderson et al [2] have discussed models for rabies with a density dependent death rate McLean and Anderson [17,18] also incorporated this feature in discussing a model of measles Gao and Hethcote [11] studied SIRS/SIS models with restricted population growth by logistic equation due to density dependence in both birth and death rates They also considered density dependent transmission coefficient (inversely proportional to the total population size) They obtained four equilibria and discussed their stability results

Mathematicians working in theoretical ecology have considered density dependence in death rate and transmission coefficient recently. One may refer to Brauer [5,6], Busenberg and Hadeler [7], 
Busenberg and Van den Driesche [8], Diekmann and Kretzschmav [9], Greenhalgh [12-14], May and Anderson [3], Pugliese [21,22] and Tuljapurkar and Merdith-John [23]

Greenhalgh [12] described a mathematical model for a disease where the death rate is a monotonically increasing function of the number of individuals in the population and infected individuals suffer a higher death rate than other individuals The same author in $[13,14]$ studied the modified model with a class of individuals who are incubating the disease and vaccination of susceptible individuals In our model we have considered density dependence in the transmission coefficient (inversely proportional to the total population) together with the vaccination of susceptible individuals Considering density dependence of the transmıssion parameter, we can relax the assumption that the number of contacts per unit time per susceptible individual increases linearly with the population size

\section{MATHEMATICAL MODEL}

We examine a model in which an individual starts as a susceptible, catches the disease and after a short infectious period becomes permanently immune to it We assume the individuals who are susceptible are vaccinated at a constant per capital rate $c$ The spread of the disease is modeled by a set of differential equations which describe the transfer of individuals between these classes The system of ordinary differential equations which describe the spread of the disease is as follows

$$
\begin{aligned}
\frac{d x}{d t} & =r N-\frac{b}{N} x y-c x-f(N) x \\
\frac{d y}{d t} & =\frac{b}{N} x y-f(N) y-(\nu+\alpha) y \\
\frac{d z}{d t} & =\nu y+c x-f(N) z \\
\frac{d N}{d t} & =r N-f(N) N-\alpha y
\end{aligned}
$$

with suitable initial conditions, where one of the equations is redundant since $x(t)+y(t)+z(t)=N(t)$

$x(t)$ represents the population (or density) of the susceptible class at time $t$,

$y(t)$ represents the population of the infected class at time $t$,

and $z(t)$ represents the population of the immune class at time $t$, i e. those individuals who have had the disease, have recovered and are permanently immune

In our model, $r$ is the birth rate, $\frac{b}{N}$ is the transmission coefficient, $f(N)$ is the density dependent death rate taken as a continuous, strictly monotonic increasing function of $N$ (Greenhalgh [12-14], considered $\frac{b}{N}=\beta=$ constant), $\alpha$ is the additional death rate suffered by infected individuals, and $\nu$ is the rate at which infected individuals become immune, so that $\nu^{-1}$ is the average infectious period in the absence of a death rate The probability that a susceptible individual meets and becomes infected by an infectious individual in $[t, t+\Delta t]$ is $\frac{b}{N}(\Delta t)+o(\Delta t)$ The per capita rate of vaccination of susceptible individuals is $c$, so that in a small time interval $[t, t+\Delta t]$ the number of susceptible individuals who are vaccinated is $c x \triangle t+o(\triangle t)$ This term $c x$ must be subtracted from the equation (2.1a) corresponding to the fraction of susceptible individuals who are vaccinated and added to equation $(2 \mathrm{lc})$ corresponding to new immune individuals. We are interested in performing an equilibrium and stability analysis of this model The stability analysis helps us to determine the long-term behavior of the system, i e whether the disease persists

\section{EQUILIBRIUM ANALYSIS.}

The first step is to examine the possible equilibrium solutions of these equations First of all, we shall suppose that $f(\infty)=\lim _{N \rightarrow \infty} f(N)>r$, so that if the population size is large enough the death rate exceeds the birth rate Setting all of the time derivative to zero in system $(21)$, we deduce the following theorem for the possible equilibrium solutions 
THEOREM Let $\widehat{x}, \widehat{y}$, and $\widehat{z}$ denote the equilibrium numbers of susceptible, infected and immune individuals respectively Let $\widehat{N}$ denote the total number of individuals at equilibrium

There are three possible equilibria

(i) When there is no disease present because the population has died out

$$
\widehat{x}=\widehat{y}=\widehat{z}=\widehat{N}=0 .
$$

(ii) If $f(\infty)>r>f(0)$, the population has reached an equilibrium level but the disease has died out

$$
\widehat{x}=\frac{f(\widehat{N}) \widehat{N}}{c+f(\widehat{N})}, \quad \widehat{y}=0, \quad \widehat{z}=\frac{c \widehat{N}}{c+f(\widehat{N})}
$$

and

$$
r=f(\widehat{N}) .
$$

(iii) The disease is present and the equilibrium values of susceptible, infected and immune individuals, are

$$
\begin{aligned}
& \widehat{x}=\frac{(\nu+\alpha+f(\widehat{N})) \widehat{N}}{b}, \quad \widehat{y}=\frac{(r-f(\widehat{N})) \widehat{N}}{\alpha}, \\
& \widehat{z}=\frac{\widehat{N}\{\nu b(r-f(\widehat{N}))+c \alpha(\nu+\alpha+f(\widehat{N}))\}}{\alpha b f(\widehat{N})} .
\end{aligned}
$$

Population value $\widehat{N}$ satisfies the equation

$$
\frac{b}{\alpha}=\frac{(f(\widehat{N})+c)(\nu+\alpha+f(\widehat{N}))}{f(\widehat{N})^{2}+(\nu+\alpha-r) f(\widehat{N})-\nu r} .
$$

This equilibrium exists if and only if $b \geq \frac{(r+c)(\nu+\alpha+r)}{r}$

PROOF. This theorem is proved along similar lines to the corresponding results for the related models in Greenhalgh [12-14] Setting the time derivatives to zero in system (2 1), we deduce that

$$
\begin{array}{r}
r \widehat{N}^{2}-b \widehat{x} \widehat{y}-c \widehat{N} \widehat{x}-\widehat{N} f(\widehat{N}) \widehat{x}=0 \\
b \widehat{x} \widehat{y}-\widehat{N} f(\widehat{N}) \widehat{y}-(\nu+\alpha) \widehat{N} \widehat{y}=0 \\
\nu \widehat{y}+c \widehat{x}-f(\widehat{N}) \widehat{z}=0 \\
r \widehat{N}-f(\widehat{N}) \widehat{N}-\alpha \widehat{y}=0
\end{array}
$$

From equation (3 6b) either $\widehat{y}=0$ or $\widehat{x}=\frac{\widehat{N} f(\widehat{N})+(\nu+\alpha) \widehat{N}}{b} \quad$ Now $\widehat{y}=0$ implies from equation (3 6d) that $\widehat{N}=0$ or $r=f(\widehat{N}) \quad$ Hence if $r \neq f(\widehat{N})$ then $\widehat{x}=\widehat{y}=\widehat{z}=\widehat{N}=0 \quad$ If $r=f(\widehat{N})$ then it is straightforward to show that equations (3 6a) and (3 6c) yield

$$
\widehat{x}=\frac{f(\widehat{N}) \widehat{N}}{c+f(\widehat{N})}, \quad \widehat{y}=0, \quad \widehat{z}=\frac{c \widehat{N}}{c+f(\widehat{N})},
$$

an equilibrium solution for any value of $\widehat{N}$ If $\widehat{x}=\frac{\widehat{N} f(\widehat{N})+(\nu+\alpha) \widehat{N}}{b}$ then from equation (3 6a) we have

$$
\widehat{y}=\frac{r \widehat{N}^{2}-c \widehat{N} \widehat{x}-\widehat{N} f(\widehat{N}) \widehat{x}}{b \widehat{x}}=\frac{r \widehat{N}}{f(\widehat{N})+\nu+\alpha}-\frac{(c+f(\widehat{N})) \widehat{N}}{b}
$$

and from equation $(36 \mathrm{~d})$ 


$$
\widehat{y}=\frac{(r-f(\widehat{N})) \widehat{N}}{\alpha}
$$

Equating these expressions for $\widehat{y}$, we get an equation for $\widehat{N}$ which can be reduced to

$$
\frac{b}{a}=\frac{(f(\widehat{N})+c)(\nu+a+f(\widehat{N}))}{f(\widehat{N})^{2}+(\nu+\alpha-r) f(\widehat{N})-\nu r} .
$$

Thus the equilibrium values $\widehat{x}, \widehat{y}, \widehat{z}$ and $\widehat{N}$ must satısfy the values given in (i), (ii) and (iii) of the theorem

The first equilibrium is always possible The second equilibrium is well defined if and only if $f(0)<r<f(\infty)$ The third equilibrium exists if and only if

$$
b \geq \frac{(r+c)(\nu+\alpha+r)}{r}
$$

Is well defined using the following lemma

LEMMA. The equation $\frac{b}{\alpha}=\frac{(f(\widehat{N})+c)(\nu+\alpha+f(\widehat{N}))}{f(\widehat{N})^{2}+(\nu+\alpha-r) f(\hat{N})-\nu r}$ has a unique positive root $\widehat{N}$, if and only if $f(\infty) \underline{\exists}_{+}$, where $\xi_{+}$is the unique positive root of $\left.Q \dot{f}\right)=0$ Here

$$
Q(\xi)=\xi^{2}+(\nu+\alpha-r) \xi-\nu r .
$$

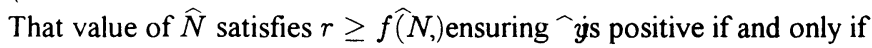

$$
b \geq \frac{(r+c)(\nu+\alpha+r)}{r} .
$$

PROOF. Consider the equation

$$
\frac{b}{\alpha}=\frac{(f(\widehat{N})+c)(\nu+\alpha+f(\widehat{N}))}{f(\widehat{N})^{2}+(\nu+\alpha-r) f(\widehat{N})-\nu r},
$$

With the transformation $\xi=f(\widehat{N})$ Then

$$
\frac{b}{\alpha}=\frac{(\xi+c)(\nu+\alpha+\xi)}{\xi^{2}+(\nu+\alpha-r) \xi-\nu r}
$$

Consider $g(\xi)=\frac{(\xi+c)(\nu+\alpha+\xi)}{\xi^{2}+(\nu+\alpha-r) \xi-\nu r}$. Here $g(\xi)$ has roots $\xi=-c,-\nu-\alpha \quad$ The asymptotes are the roots of $Q(\xi)=0$, where

$$
Q(\xi)=\xi^{2}+(\nu+\alpha-r) \xi-\nu r .
$$

$Q(\xi)$ has two real roots $\xi_{-}$and $\xi_{+}$given by

$$
\xi_{-}, \xi_{+},=-\frac{1}{2}(\alpha+\nu-r) \pm \frac{1}{2}\left[(\alpha+\nu-r)^{2}+4 \nu r\right]^{\frac{1}{2}} .
$$

The function $g(\xi)$ is negative for $0 \leq \xi<\xi_{+}$and monotonically decreasing for $\xi>\xi_{+}$. Hence the equation

$$
\frac{b}{\alpha}=g(\xi)
$$

has a unique positive root $\xi$ if and only if $f(\infty)>\xi_{+}$Otherwise, if $f(\infty) \leq \xi_{+}$this equation has no positive root for $\xi$ Since $f(\infty)>\xi_{+}$denote the unique root by $\widehat{\xi}$ and let $\widehat{N}$ be the corresponding value of $N$ For the solution to be feasible we require $f(\widehat{N}) \leq r$ or $\widehat{\xi} \leq r$ as $\frac{b}{\alpha}$ is constant and $g(\xi)$ is monotonically decreasing in $\xi$, it follows that 
is monotonically increasing in $\xi$ and zero at $\widehat{\xi}$ [see the graph of $g(\xi)$ ] Hence $r \geq \widehat{\xi}$ if and only if

$$
\frac{b}{\alpha}-g(r) \geq 0 \text {. }
$$

This condition is equivalent to

$$
b \geq \frac{(r+c)(\nu+\alpha+r)}{r}
$$

This completes the proof of the lemma

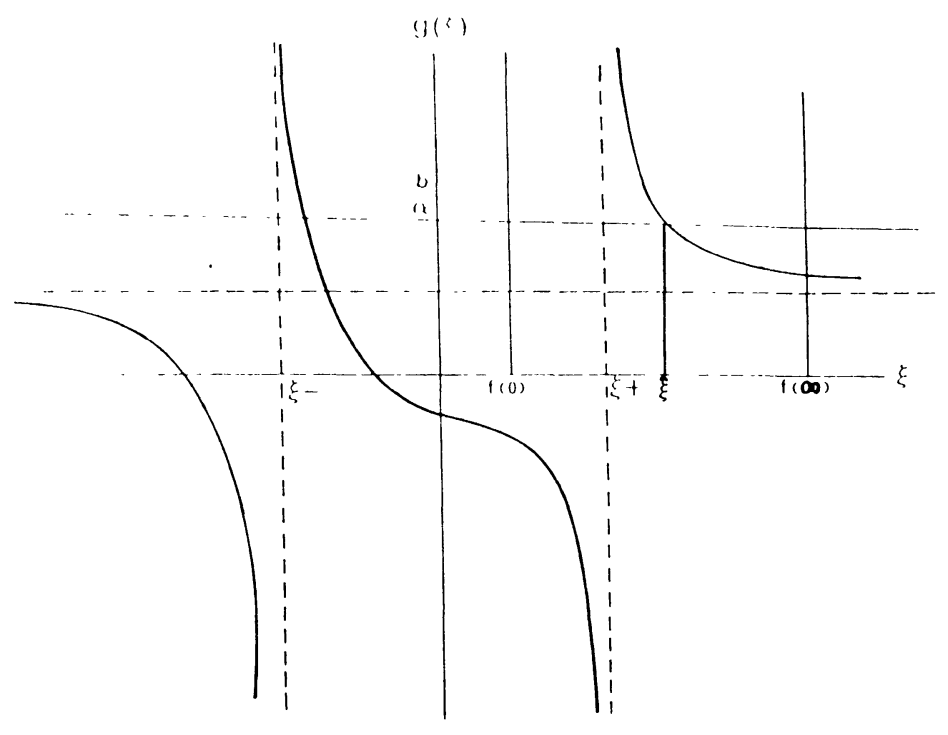

(ira)h if ( ) ( 5$)$

\section{STABILITY}

\section{Stability Analysis of Equilibrium (i).}

By Liapunov's (Jordan and Smith [15]) indirect method we determine the stability behavior of the system of differential equations $(21)$ which describe the spread of the disease at the equilibrium $\widehat{x}=\widehat{y}=\widehat{z}=\widehat{N}=0 \quad$ Consider the Liapunov function $L=x+y+z$ which leads to

$$
L^{\prime}=(r-f(N)) N-\alpha y<(r-f(0)) N-\alpha y \leq 0 \text { when } \quad N>0 \quad \text { and } \quad r \leq f(0) .
$$

We conclude that the zero solution of $(21)$ is globally asymptotic stable (GAS) for $r \leq f(0)$ since $L^{\prime}<0$ and unstable for $r>f(0)$. Therefore it follows that the zero solution of the original system (2 1$)$ is GAS for $r \leq f(0)$ and unstable for $r>f(0)$

\section{Analysis of Equilibrium (ii).}

The equilibrium values are

$$
\widehat{x}=\frac{f(\widehat{N}) \widehat{N}}{c+f(\widehat{N})}, \quad \widehat{y}=0, \quad \widehat{z}=\frac{c \widehat{N}}{c+f(\widehat{N})} .
$$

Consider a small perturbation about this equilibrium level

$$
x=\widehat{x}+x_{1}, \quad y=\widehat{y}+y_{1}, \quad z+\widehat{z}+z_{1} \quad \text { and } \quad N=\widehat{N}+n_{1} .
$$

Substituting these into the differential equations which describe the spread of the disease, and using the approximation $f\left(\widehat{N}+n_{1}\right)=f(\widehat{N})+n_{1} f^{\prime}(\widehat{N})+o\left(n_{1}\right)$ we get the stability matrix $A$ as 
Substituting these into the differential equations which describe the spread of the disease, and using the approximation $f\left(\widehat{N}+n_{1}\right)=f(\hat{N})+n_{1} f^{\prime}(\widehat{N})+o\left(n_{1}\right)$ we get the stability matrix $A$ as

$$
A=\left(\begin{array}{cccc}
-(c+f(\hat{N})) & -\frac{1 \hat{i}}{\hat{N}} & 0 & f(\widehat{N})-f^{\prime}(\widehat{N}) \widehat{x} \\
0 & \frac{b \hat{r}}{\hat{N}^{\prime}}-(f(\widehat{N})+\nu+\alpha) & 0 & 0 \\
c & \nu & -f(\widehat{N}) & -f^{\prime}(\widehat{N}) \widehat{z} \\
0 & -\alpha & 0 & -f^{\prime}(\widehat{N}) \widehat{N}
\end{array}\right)
$$

which gives the characteristic equation as

$$
(f(\widehat{N})+\lambda)(c+f(\widehat{N})+\lambda)\left\{\frac{b \widehat{x}}{\widehat{N}}-(f(\widehat{N})+\nu+\alpha)-\lambda\right\}\left(f^{\prime}(\widehat{N}) \widehat{N}+\lambda\right)=0 .
$$

From equations (3 2), ( 33 ) and (4.3) we get the equilibrium (ii) to be locally stable for small perturbations if $R_{0}<1$ and locally unstable if $R_{0}>1$ where

$$
R_{0}=\frac{b r}{(c+r)(r+\nu+\alpha)} .
$$

\section{Local Stability of Equilibrium (iii).}

Using the same procedure as for equilibrium (ii), we get the stability matrix $C$ as follows

$$
C=\left(\begin{array}{cccc}
-\frac{b \widehat{y}}{\hat{N}}-c-f(\widehat{N}) & -\frac{b \widehat{x}}{\widehat{N}} & 0 & r+\frac{b \widehat{x} \widehat{y}}{\widehat{N}^{2}}-f^{\prime}(\widehat{N}) \widehat{x} \\
\frac{b \hat{y}}{\widehat{N}} & 0 & 0 & -f^{\prime}(\widehat{N}) \widehat{y}-\frac{b \widehat{x} \widehat{y}}{\widehat{N}^{2}} \\
c & \nu & -f(\widehat{N}) & -f^{\prime}(\widehat{N}) \widehat{z} \\
0 & -\alpha & 0 & r-f(\widehat{N})-f^{\prime}(\widehat{N}) \widehat{N}
\end{array}\right) .
$$

The determinant of $C-\lambda I$ is $|C-\lambda I|=-(f(\widehat{N})+\lambda)|D|$, where

$$
D=\left(\begin{array}{ccc}
-\frac{b \hat{y}}{\hat{N}}-c-f(\widehat{N})-\lambda & -\frac{b \widehat{x}}{\hat{N}} & r+\frac{b \widehat{x} \hat{y}}{\widehat{N}^{2}}-f^{\prime}(\widehat{N}) \widehat{x} \\
\frac{b \hat{y}}{\hat{N}} & -\lambda & -f^{\prime}(\widehat{N}) \widehat{y}-\frac{b \widehat{x y}}{\widehat{N}^{2}} \\
0 & -\alpha & r-f(\widehat{N})-f(\widehat{N}) \widehat{N}-\lambda
\end{array}\right) .
$$

The corresponding characteristic equation for $D$ is

$$
(f(\widehat{N})+\lambda)\left(\lambda^{3}+a_{1} \lambda^{2}+a_{2} \lambda+a_{3}\right)=0,
$$

where

$$
\begin{aligned}
a_{1}= & \left(\frac{b \widehat{y}}{\widehat{N}}+c+2 f(\widehat{N})-r\right)+f^{\prime}(\widehat{N}) \widehat{N}, \\
a_{2}= & \left\{-\frac{b \widehat{y}}{\widehat{N}}(r-f(\widehat{N}))-r f(\widehat{N})+f^{2}(\widehat{N})-c(r-f(\widehat{N}))-\frac{\alpha b \widehat{x} \widehat{y}}{\widehat{N}^{2}}+\frac{b^{2} \widehat{x} \widehat{y}}{\widehat{N}^{2}}\right\} \\
& +f^{\prime}(\widehat{N})\{b \widehat{y}+(c+f(\widehat{N})) \widehat{N}-\alpha \widehat{y}\}, \\
a_{3}= & \left\{-\frac{\alpha b \widehat{x} \widehat{y}}{\widehat{N}^{2}}\left(\frac{b \widehat{y}}{\widehat{N}}+f(\widehat{N})\right)+\frac{\alpha b r \widehat{y}}{\widehat{N}}-\frac{\alpha b \widehat{x} \widehat{y}}{\widehat{N}^{2}} c\right\} \\
& +f^{\prime}(\widehat{N})\left\{-\alpha \widehat{y}\left(\frac{b \widehat{y}}{\widehat{N}}+f(\widehat{N})\right)+\frac{b^{2} \widehat{x} \widehat{y}}{\widehat{N}}-\frac{\alpha b \widehat{x} \widehat{N}}{\widehat{N}}-\alpha c \widehat{y}\right\} .
\end{aligned}
$$

The Routh-Hurwitz (May [16]) stability criteria for the third order system is

$$
\begin{aligned}
& \text { (i) } a_{1}>0, a_{2}>0 \text { and } a_{3}>0 \text {; } \\
& \text { (ii) } a_{1} a_{2}>a_{3} .
\end{aligned}
$$


Now we will show the positivity of all the constants appearing in (4 8)

Let

$$
\begin{aligned}
& u_{1}=\frac{b \widehat{y}}{\widehat{N}}+c+2 f(\widehat{N})-r \\
& u_{2}=\widehat{N} \\
& v_{1}=-\frac{b \widehat{y}}{\widehat{N}}(r-f(\widehat{N}))-r f(\widehat{N})+f^{2}(\widehat{N})-c(r-f(\widehat{N}))-\frac{\alpha b \widehat{x y}}{\widehat{N}^{2}}+\frac{b^{2} \widehat{x y}}{\widehat{N}^{2}} \\
& v_{2}=b \widehat{y}+(c+f(\widehat{N})) \widehat{N}-\alpha \widehat{y}, \\
& w_{1}=-\frac{\alpha b \widehat{x} \widehat{y}}{\widehat{N}^{2}}\left(\frac{b \widehat{y}}{\widehat{N}}+f(\widehat{N})\right)+\frac{\alpha b r \widehat{y}}{\widehat{N}}-\frac{\alpha b \widehat{x} \hat{y} c}{\widehat{N}^{2}} \\
& w_{2}=-\alpha \widehat{y}\left(\frac{b \widehat{y}}{\widehat{N}}+f(\widehat{N})\right)+\frac{b^{2} \widehat{x} \widehat{y}}{\widehat{N}}-\frac{\alpha b \widehat{x} \widehat{y}}{\widehat{N}}-\alpha c \widehat{y} .
\end{aligned}
$$

Rewriting equation (4 8) as

$$
\begin{aligned}
& a_{1}=u_{1}+f^{\prime}(\widehat{N}) u_{2}, \\
& a_{2}=v_{1}+f^{\prime}(\widehat{N}) v_{2}, \\
& a_{3}=w_{1}+f^{\prime}(\widehat{N}) w_{2} .
\end{aligned}
$$

Since $f(N)$ is monotonic increasing in $N, f^{\prime}(\widehat{N}) \geq 0$ Hence to show (i) of equation (49) we will show that $u_{1}, u_{2}, v_{1}, v_{2}, w_{1}$ and $w_{2}$ are all positive

Using equation (3 5) we get

$$
u_{1}=(r-f(\widehat{N}))\left(\frac{b-\alpha}{\alpha}\right)+f(\widehat{N})+c,
$$

where $r>f(\widehat{N})$ and from equation (3 4) $b>\alpha$, therefore $u_{1}>0$

Then since $u_{2}=\widehat{N}>0$, it must be that $a_{1}>0$

Now $v_{1}$ can be rewritten as

$$
\begin{aligned}
v_{1}=-(r-f(\widehat{N}))\left\{\frac{b}{\alpha}(r-f(\widehat{N}))+f(\widehat{N})+c\right\} & -(r-f(\widehat{N}))(\nu+\alpha+f(\widehat{N})) \\
& +\frac{b}{\alpha}(r-f(\widehat{N}))(\nu+\alpha+f(\widehat{N})) .
\end{aligned}
$$

Now $v_{1}>0$ if

$$
-\frac{b}{\alpha}(r-f(\widehat{N}))-(f(\widehat{N})+c)-(\nu+\alpha+f(\widehat{N}))+\frac{b}{\alpha}(\nu+\alpha+f(\widehat{N}))>0 .
$$

With the help of equation ( 34 ), the above inequality reduces to the following form

$$
\begin{aligned}
&-\frac{b}{\alpha}(r-f(\widehat{N}))- \frac{b}{\alpha}\left\{\frac{f(\widehat{N})(f(\widehat{N})+\nu+\alpha)-r(f(\widehat{N})+\nu)}{(\nu+\alpha+f(\widehat{N}))}\right\} \\
&-(\nu+\alpha+f(\widehat{N}))+\frac{b}{\alpha}(\nu+\alpha+f(\widehat{N}))>0 . \\
& \text { or } \quad \frac{(\nu+\alpha+f(\widehat{N}))^{2}(b-\alpha)-b r \alpha}{\alpha(f(\widehat{N})+\nu+\alpha)}>0 .
\end{aligned}
$$

Hence

$$
v_{1}>0 \quad \text { if } \quad(\nu+\alpha+f(\widehat{N}))^{2}(b-\alpha)>b r \alpha .
$$

From equation (3 4) 


$$
\frac{b-a}{b}>\frac{r a}{(\nu+a+f(\hat{N}))(r-f(\hat{N}))} .
$$

Since $\hat{y}<\hat{N}$, from equation (6) $r-f(\hat{N})<\alpha$, so that

$$
r-f(\widehat{N})<a+\nu+f(\widehat{N}) .
$$

Inequalities (4 14) and (4 15) combined to show that the inequality (4 13) is true i.e $v_{1}>0$

Now

$$
v_{2}>0 \quad \text { if } \quad b \widehat{y}+\widehat{N} f(\widehat{N})-\alpha \widehat{y}+c \widehat{N}>0
$$

or

$$
(b-\alpha) \widehat{y}+\widehat{N} f(\widehat{N})+c \widehat{N}>0
$$

From equation ( 34$), b>\alpha$, therefore the above inequality is true and hence $a_{2}>0$

$$
w_{1}=\frac{\alpha b \widehat{y}}{\widehat{N}}\left(-\frac{b \widehat{x} \widehat{y}}{\widehat{N}^{2}}-\frac{\widehat{x} f(\widehat{N})}{\widehat{N}}+r-\frac{\widehat{x} c}{\widehat{N}}\right) .
$$

Using equation ( 35 ) the terms inside the bracket can be written as

$$
\begin{aligned}
& -\frac{b \widehat{x y}}{\widehat{N}^{2}}-\frac{\widehat{x} f(\widehat{N})}{\widehat{N}}+r-\frac{\widehat{x} c}{\widehat{N}} \\
& =-\frac{(\nu+\alpha+f(\widehat{N}))(r-f(\widehat{N}))}{\alpha} \\
& -\frac{(\nu+\alpha+f(\widehat{N})) f(\widehat{N})}{b}+r-\frac{c(\nu+\alpha+f(\widehat{N})}{b} \\
& =\frac{1}{b \alpha}\left\{-\alpha(\nu+\alpha+f(\widehat{N}))(f(\widehat{N})+c)+b f^{2}(\widehat{N})+b f(\widehat{N})(\nu+\alpha-r)-b r \nu\right\} \\
& =0 .
\end{aligned}
$$

Thus $w_{1}=0$

$$
\begin{aligned}
w_{2} & =-\alpha \widehat{y}\left(\frac{b \widehat{y}}{\widehat{N}}+f(\widehat{N})\right)+\frac{b^{2} \widehat{x} \widehat{y}}{\widehat{N}}-\frac{\alpha b \widehat{x} \widehat{y}}{\widehat{N}}-\alpha c \widehat{y} \\
& =\widehat{y}\left\{-\frac{\alpha b \widehat{y}}{\widehat{N}}-\alpha f(\widehat{N})+\frac{b^{2} \widehat{x}}{\widehat{N}}-\frac{\alpha b \widehat{x}}{\widehat{N}}-\alpha c\right\} .
\end{aligned}
$$

Now $w_{2}>0$ if the sum of all terms inside the bracket is positive. Using equation ( 35 ), the terms inside the bracket become

$$
\alpha\left\{-\frac{b}{\alpha}(r-f(\widehat{N}))-(f(\widehat{N})+c)+\frac{b}{\alpha}(\nu+\alpha+f(\widehat{N}))-(\nu+\alpha+f(\widehat{N}))\right\} .
$$

We have already shown above that the sum of all terms of $(416)$ is positive

Hence, $a_{3}>0$

Now we have to show that $a_{1} a_{2}-a_{3}>0$.

$$
a_{1} a_{2}-a_{3}=\left(u_{1} v_{1}-w_{1}\right)+f^{\prime}(\widehat{N})\left(u_{1} v_{2}+u_{2} v_{1}-w_{2}\right)+{f^{\prime}}^{2}(\widehat{N})\left(u_{2} v_{2}\right),
$$

where $w_{1}=0$ and $u_{1}, u_{2}, v_{1}, v_{2}, w_{2}$ all are positive i.e. to show $a_{1} a_{2}-a_{3}>0$ we will show that $u_{1} v_{2}+u_{2} v_{1}-w_{2}>0$ We have $u_{2} v_{1}=w_{2}$ hence the inequality 


\section{SUMMARY AND CONCLUSIONS}

In this paper we have studied a simple mathematical epidemic model with vaccination There are three possible equilibrium situations which arise Equilibrium with population extinct will be globally asymptotically stable if $r \leq f(0)$ and will be unstable if $r>f(0)$ Equilibrium with steady population and no disease present will be locally stable to small perturbations if $\frac{b_{r}}{(c+r)(r+\nu+\alpha,}<1$ and locally unstable If $\frac{b r}{i c+r i(r+\nu \cdot \alpha)}>1$ Local stabllity of the equilibrium with disease present is examined analytically and is found stable In this paper we have extended the work of Greenhalgh [12-14] It is unrealistic to consider the transmission parameter a constant because this assumes contact per unit time per susceptible individual increases linearly with the population size This assumption can be relaxed by taking a density dependent transmission parameter Here we consider the transmission coefficient as inversely proportional to the total number of individuals in the population Gao and Hethcote [11] have also considered density dependence in transmission coefficient similar to ours but they have taken density dependent restricted growth due to a decreasing birth rate and an increasing death rate as the population size increases towards its carrying capacity They have obtained four equilibria The first equilibrium when disease fades out and population size tends to zero and showed that it is always a saddle The second equilibrium where the population size is up the carrying capacity is found to be GAS under certain threshold conditions The third equilibrium occurs when the birth rate is density independent and the death rate is density dependent and is LAS when certain conditions are met The fourth equilibrium is achieved when the birth rate is density dependent and the death rate is density independent and it is also LAS under different threshold conditions The SIRS models reduce to SIS models when the immunity loss rate is zero All the stability results of SIRS models hold good for SIS models provided there is some inflow into the susceptible class We can make our model more realistic by the introduction of a class of individuals who are incubating the disease and taking into account the fact that immunity may only provide temporary protection

\section{REFERENCES}

[1] ANDERSON, R M, The persistence of direct life cycle infectious diseases within population of hosts, Lectures on Mathematics in the Life Sciences, Vol 12, providence, RI American Mathematical Society (1979), 1-67

[2] ANDERSON, R M, JACKSON, H C , MAY, R M and SMITH, A M, Population dynamics of fox rabies in Europe, Nature 289 (1981), 765-771

[3] ANDERSON, R.M and MAY, R M , Infectious Diseases of Humans: Dynamics and Control, Oxford University Press (1991)

[4] BAILEY, N.T J, The Mathematical Theory of Infectious Diseases and its Applications, 2nd ed, Griffin and Co London (1975).

[5] BRAUER, F., Models for the spread of universally fatal diseases, J. Math. Bıol. 28 (1990), 451462

[6] BRAUER, F, Models for the spread of universally fatal diseases II In Differential equation models in biology, epidemiology and ecology, S Busenberg and M Martelli eds, Proceedings of the International Conference in Claremont, Jan 1990, Lect. Notes Biomath. 92, Springer-Verlag (1991)

[7] BUSENBERG, S and HADELER, K P, Demography and epidemics, Math. Bloscl. 101 (1990), 63-74

[8] BUSENBERG, $S$ and VAN DEN DRIESSCHE, $P$, Analysis of a disease transmission model with varying population size, J. Math. Biol. 29 (1990), 257-270

[9] DIEKMAN, O and KRETSCHMANV, M, Patterns in the effects of infectious diseases on population growth, Report AM-R9004, February 1990, Department of Analysis, Algebra and 
Geometry, Centre of Mathematics and Computer Science, Stichting Mathematical Center, Amsterdam (1990)

[10] DIETZ, $\mathrm{K}$ and SCHENZLE, D, Proportionate mixing for age-dependent infection transmission, $J$. Math. Bıol. 22 (1985), 117-120

[11] GAO, LQ and HETHCOTE, HW, Disease transmission models with density-dependent demographics, J. Math Bıol. 30 (1992), 717-731

[12] GREENHALGH, D, An epidemic model with a density-dependent death rate, IMA J. Math. Appl. Med. Bıol. 7 (1990), 1-26

[13] GREENHALGH, D, Vaccination in density-dependent epidemic models, Bull. Math. Bıol. 54 (1992), 733-758

[14] GREENHALGH, D, Some results for an SEIR epidemic model with density-dependence in the death rate, IMA J. Math. Appl. Med. Bıol. 9 (1992), 67-106

[15] JORDAN, D W and SMITH, P, Nonlinear Ordinary Differential Equation, Clarendon press, Oxford (1987)

[16] MAY, R M, Stabllity and Complexity in Model Ecosystems, Princeton University Press (1987)

[17] McLEAN, A R and ANDERSON, R.M, Measles in developing countries, Part I Epidemiological parameters and patterns, Epidemial. Infection 100 (1988), 111-133

[18] McLEAN, A R and ANDERSON, R M, Measles in developing countries, Part II: The predicted impact of mass vaccination, Epidemial. Infection 100 (1988), 419-442.

[19] MOLLISON, D., Sensitivity analysis of simple epidemic models, In Population Dynamics of Wildlife, C P J Bacon, ed. (1985), 223-234

[20] NISBET, R.M and GURNEY, W.S.C., Modelling Fluctuatıng Populatıons, New York. Wiley (1982)

[21] PUGILESE, A, Population models for diseases with no recovery, J. math. Biol. 28 (1990), 65-82.

[22] PUGILESE, A., An SEI epidemic model with varying population size, In: Differential equation models in biology, epidemiology and ecology, S Busenberg and M Martelli eds, Proceedings of the International Conference in Claremont, Jan. 1990, Lect. Notes Biomath. 92, Springer-Verlag (1991)

[23] TULJAPURKAR, S and MERDITH-JOHN, A, Disease in changing populations. growth and disequilibrium, Theoret. Popn. Biol. 40 (1991), 322-353 


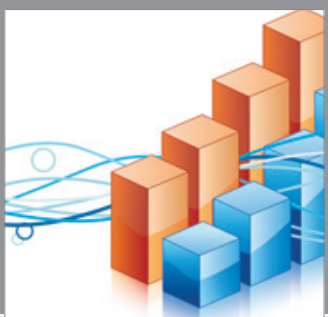

Advances in

Operations Research

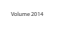

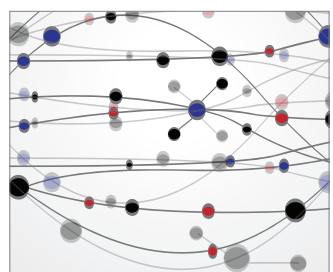

\section{The Scientific} World Journal
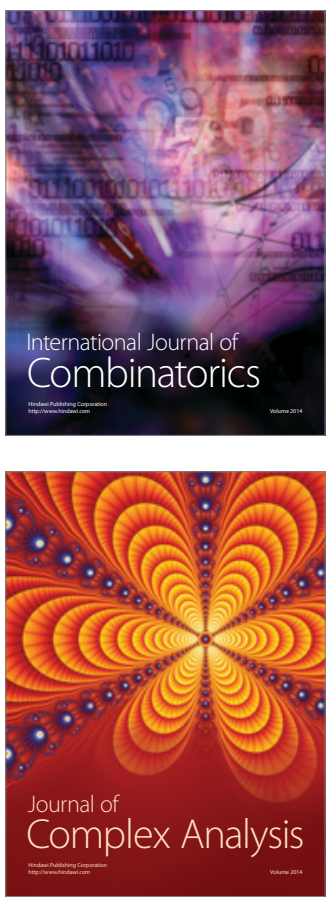

International Journal of

Mathematics and

Mathematical

Sciences
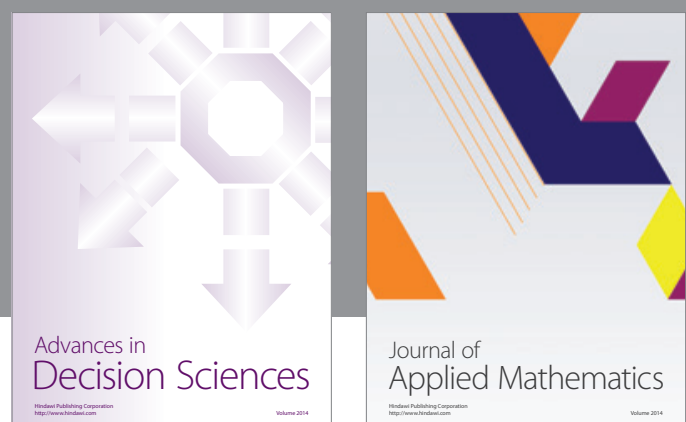

Journal of

Applied Mathematics
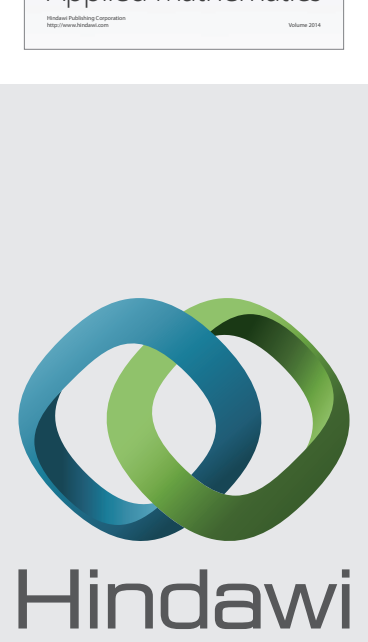

Submit your manuscripts at http://www.hindawi.com
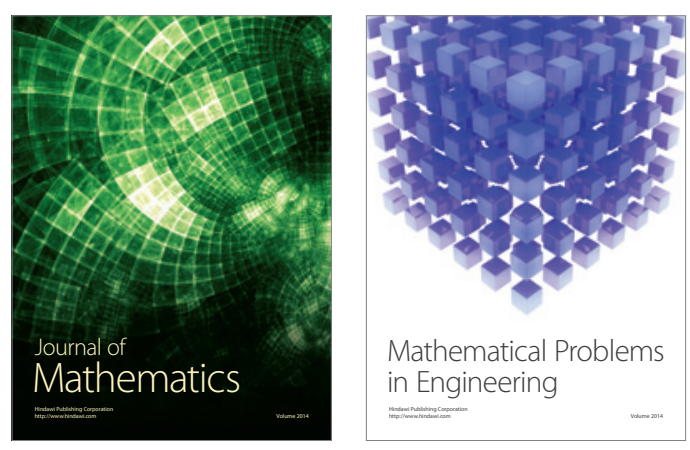

Mathematical Problems in Engineering
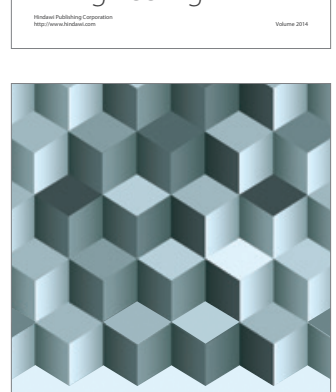

Journal of

Function Spaces
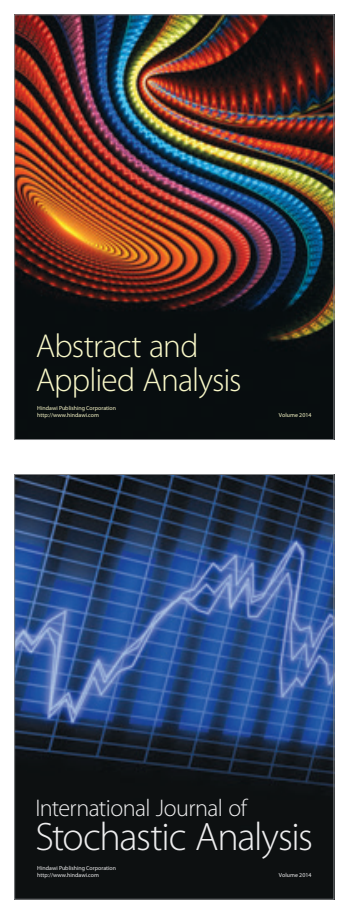

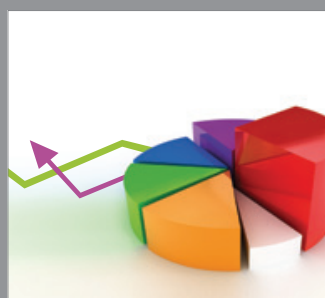

ournal of

Probability and Statistics

Promensencen
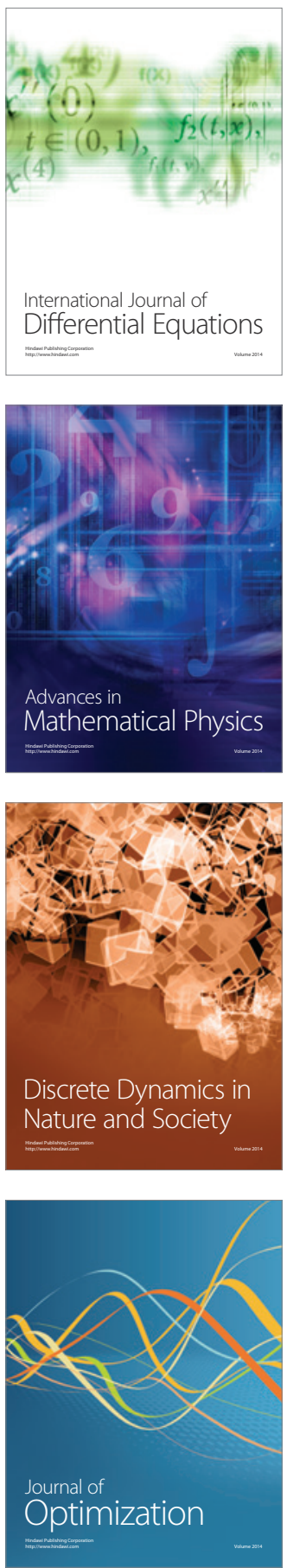